\title{
A rare ophthalmic condition associated with primary hyperparathyroidism (Scleralchoroidal Calcification)
}

Dr Mona Abouzaid*, Mr Ibrahim Masri^, Dr Satish Artham*, Mr Ajay Kotagiri^^, Dr Ashwin Joshi** *Specialist Registrar and ** Consultant, Department of Diabetes and Endocrinology Sunderland Royal City Hospitals Sunderland NHS Hospital, ^Specialist Registrar and ^^ Consultant Ophthalmologist, Sunderland Eye Infirmary.

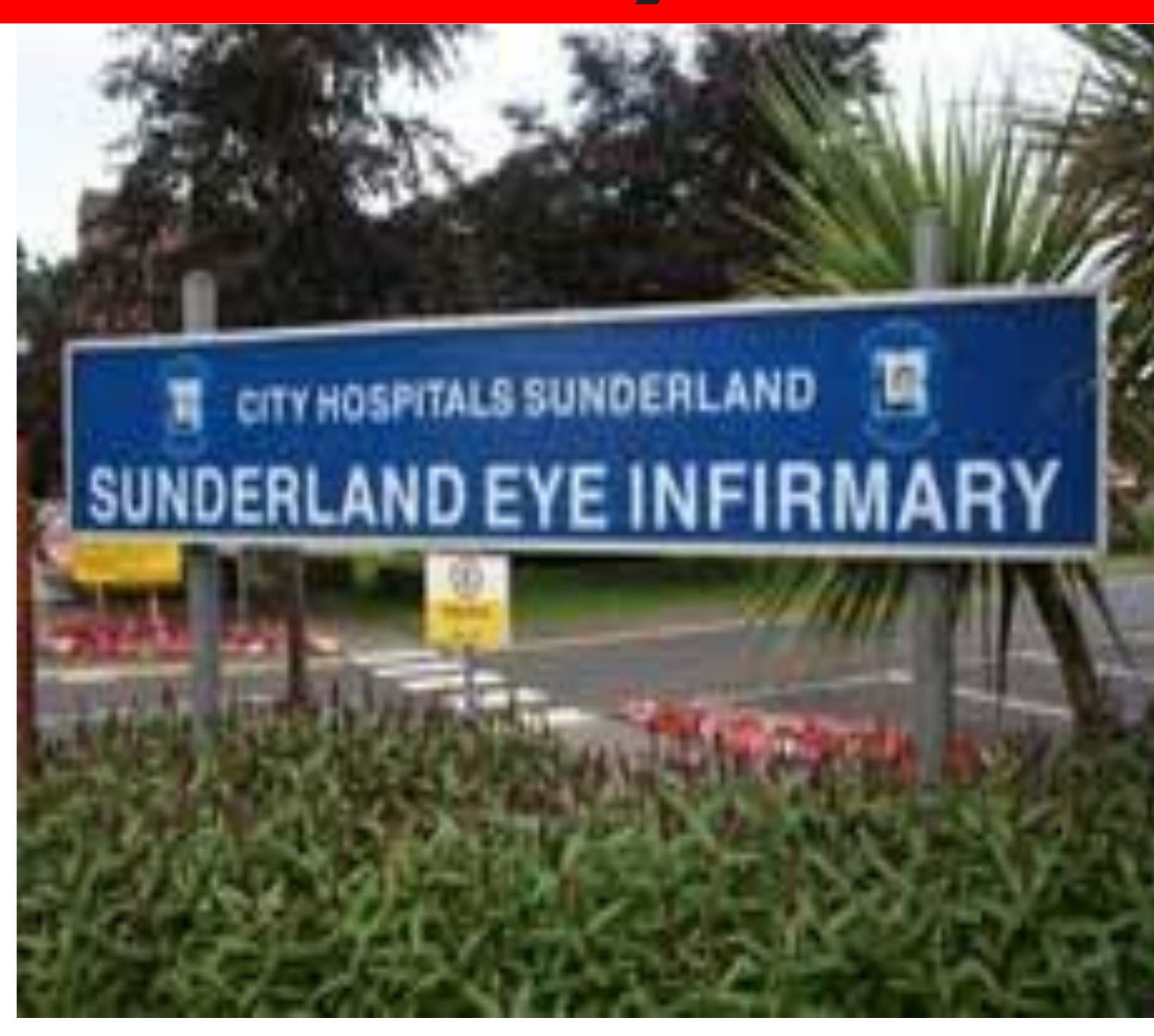

\section{Introduction}

Scleralchoroidal calcification is an uncommon condition often discovered as an incidental finding. It is ordinarily believed to be idiopathic, but is also associated with PHPT. It is important that these patients are identified because of the systemic implications and treatable nature of these disorders.

Case

82 years old patient with Primary hyperparathyroidism (PHPT) and past medical history of osteoporosis, Dyslipidemia, osteoarthritis and mild cognitive impairment was referred by the optometrist to the ophthalmology department after noticing some pale lesions in both fundi. The appearance was deemed classical of Scleralchorodial Calcification related to hypercalcaemia caused by primary hyperparathyrodism.

Right Left

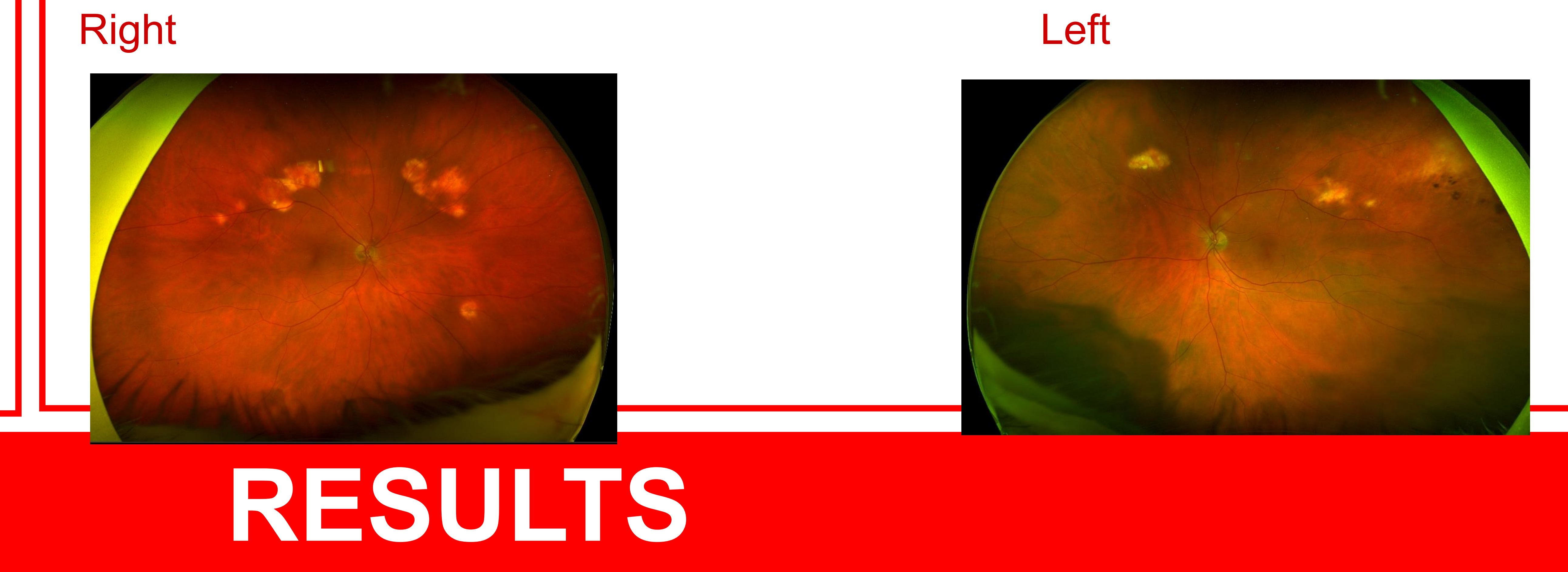

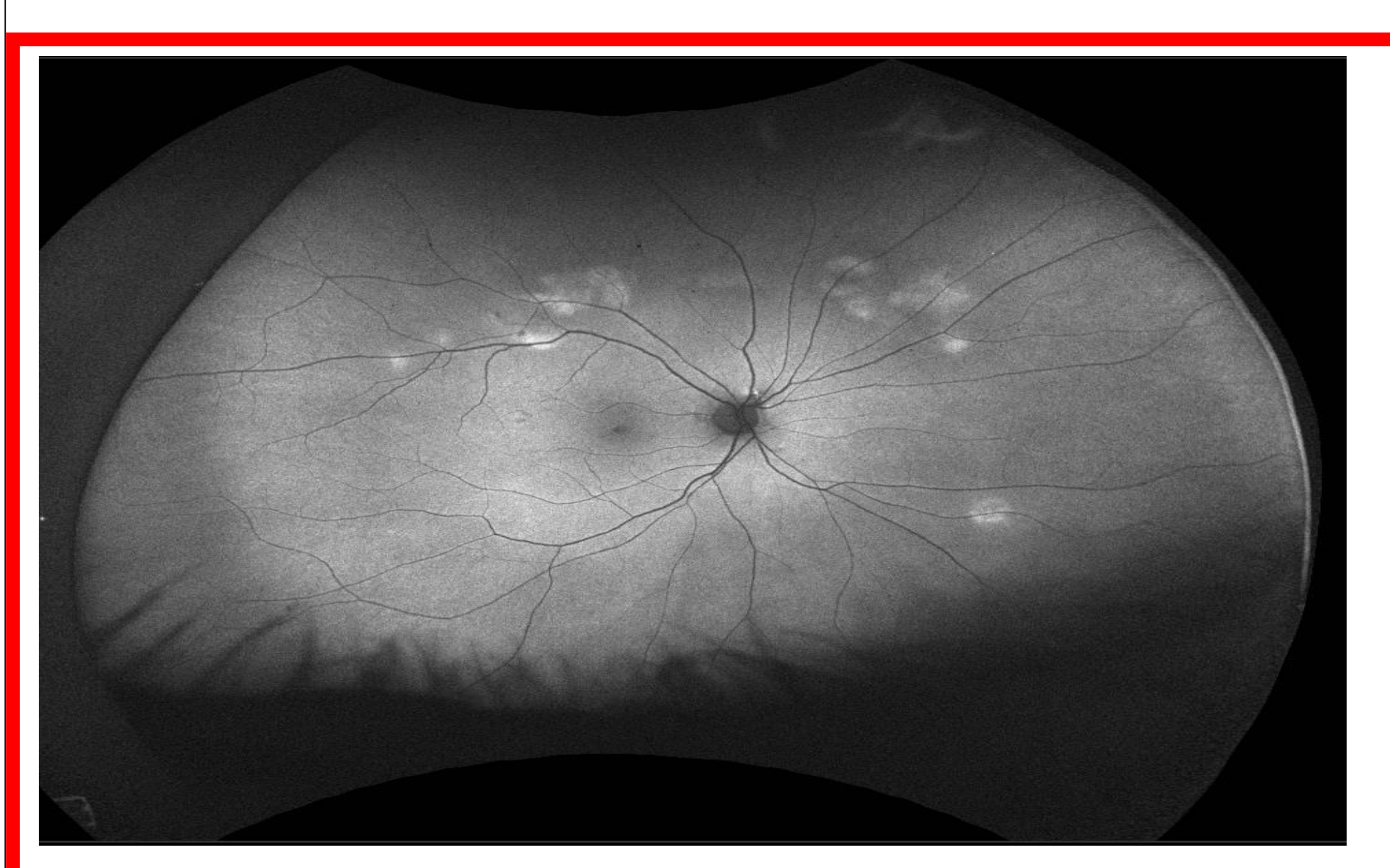

Autofluorescence photographs of his fundi showed areas of hyper-autofluorenscence corresponding with the pale lesions seen on the color pictures.

His ultrasound B-scan of both eyes shows multiple hyperechoic deposits in the posterior ocular coats -persisting at low gain. These are causing significant shadowing consistent with calcium deposits. The lesions appear to be posterior to the muscle insertion. There is no associated retinal detachment.

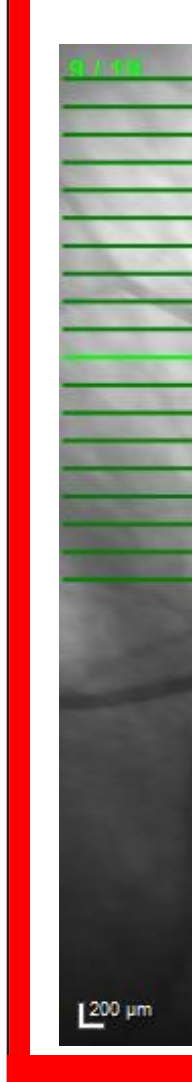

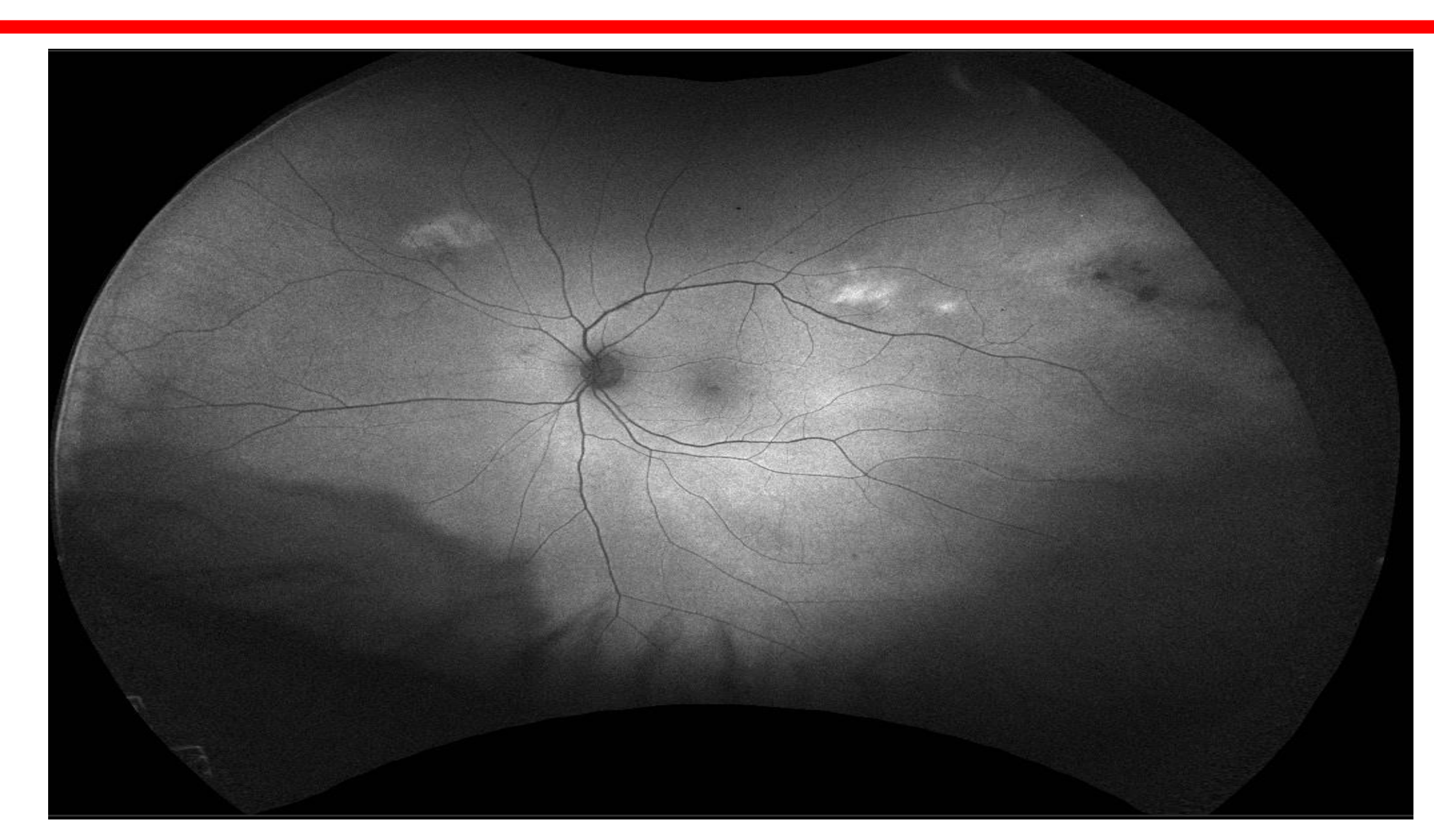

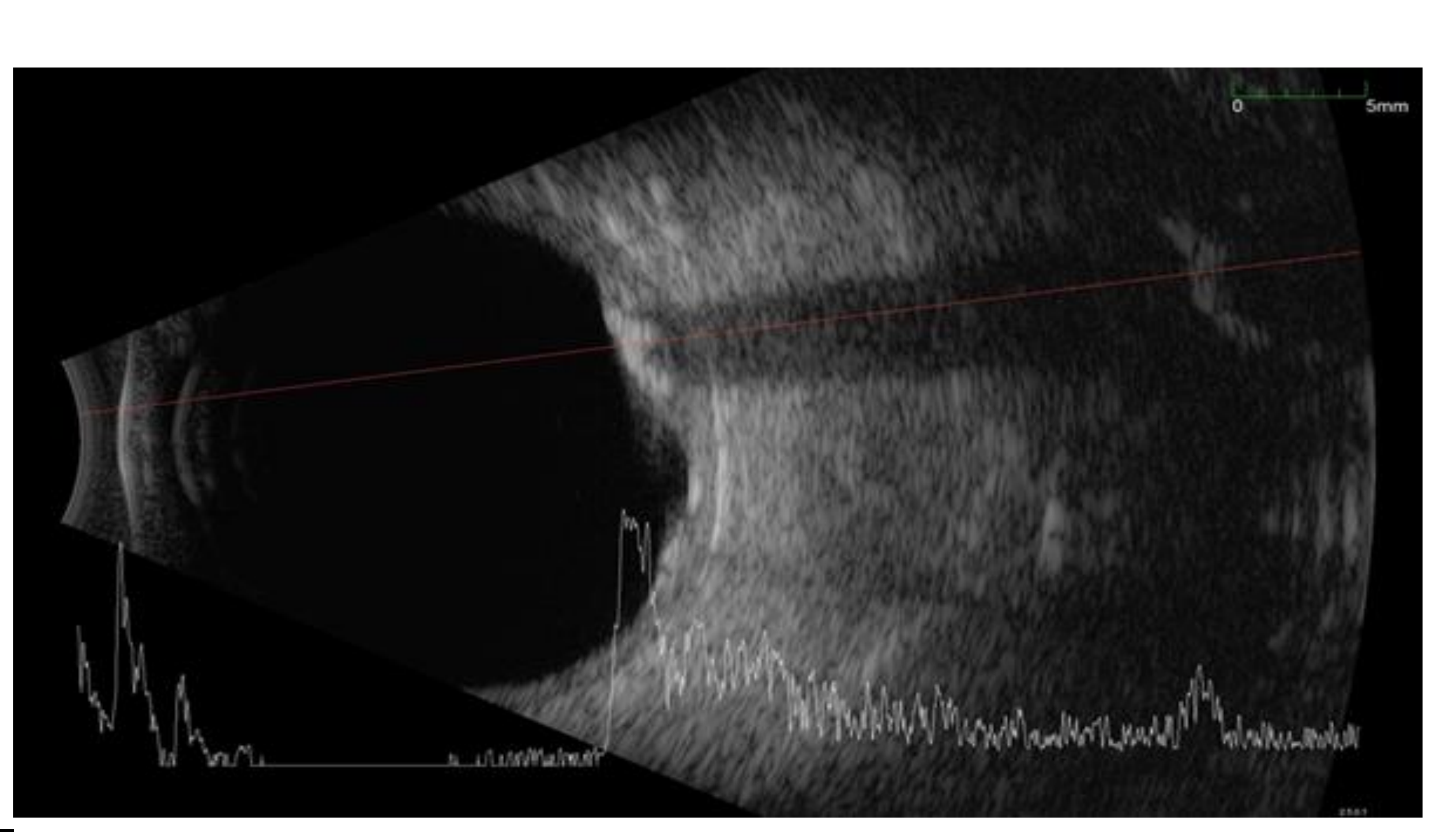

Optical Coherence Tomography (OCT) scanning through the areas of calcification (here at the level of the supratemporal area of the right eye) confirms that the abnormality is subretinal and located at the level of the choroid.
Corrected calcium

Parathyroid hormone

Phosphate level

Vitamin D

Creatinine

eGFR

Urinary calcium creatinine ratio

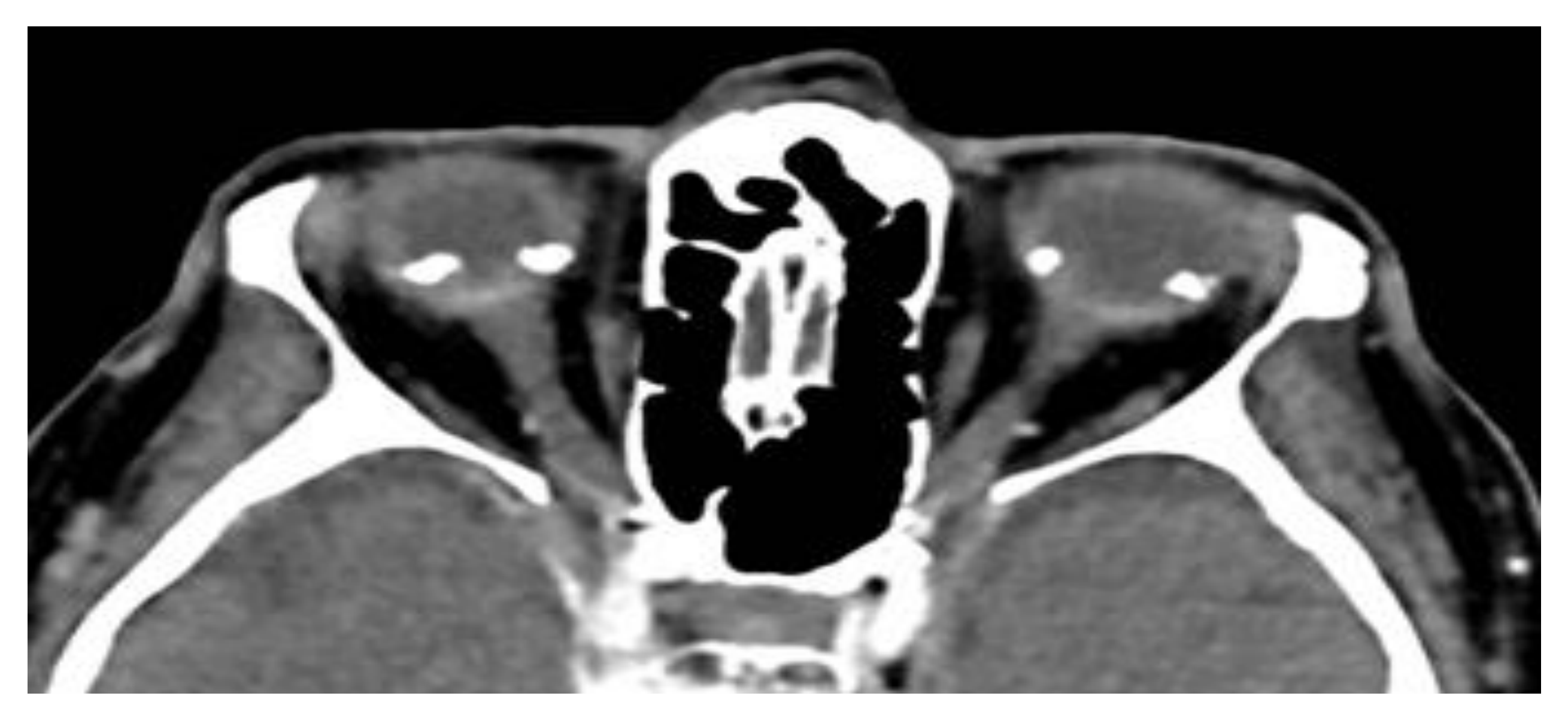

CT scan of the orbit shows bilateral sclero-choroidal calcification not involving the extraocular muscles

\section{CONCLUSIONS}

1- Collaboration between different specialties (in this case between Endocrinology and Ophthalmology) is required in managing patients. Identifying these lesions promptly helps with the management of underlying systematic disorders involving abnormal calcium - phosphorus metabolism or renal tubular hypokalemic metabolic alkalosis syndromes. All patients with sclerochoroidal calcification should be tested for these treatable systemic associations. ${ }^{1}$

2- As an Endocrinologist, it is important to look for such associations and undertake a thorough clinical examination, including fundoscopy, followed by prompt Ophthalmology referral. Our patient was already under Endocrine clinic follow up for conservative treatment of Primary Hyperparathyroidism.

3- Despite several cases of Sclerochoroidal calcification reported in the literature it remains poorly recognized and can be misdiagnosed as a choroidal metastasis, amelanotic choroidal nevus, melanoma or choroidal lymphoma resulting in unwarranted intervention ${ }^{1}$. Fluorescein angiography and ultrasonography may help in confirming the diagnosis. 